\title{
3. JAHRESTAGUNG DES ARBEITSKREISES FÜR ÜBERSEEISCHE VERFASSUNGSVERGLEICHUNG
}

Immer wenn ein neuer Lehrstuhl für Rechtsvergleichung eingerichtet oder die entsprechenden Mittel für ein ganzes Institut in diesem Forschungsbereich bewilligt werden, so läßt das jeden, der sich mit vergleichendem Verfassungsrecht beschäftigt, aufmerken. In aller Regel folgt die Enttäuschung jedoch auf dem Fuße: Die neue wissenschaftliche Einrichtung beschränkt sich nach klassischer Manier auf die Privatrechtsvergleichung, das öffentliche Recht und speziell das Verfassungsrecht anderer Staaten bleiben als Forschungsgegenstände jedenfalls im deutschsprachigen Raum weithin ausgespart. Dies war einer der bedauerlichen Befunde, zu denen der Herausgeber dieser Zeitschrift bei seinem einleitenden Bericht zum Stand der Verfassungsvergleichung auf der 3. Jahrestagung ${ }^{1}$ des Arbeitskreises für Überseeische Verfassungsvergleichung in Lüdenscheid (4.-6. 5. 1978) gelangte. Insofern kommt diesem Arbeitskreis nach wie vor eine wichtige Funktion zu; auch wenn er sich überwiegend aus „Freizeitwissenschaftlern“ zusammensetzt, die sich nebenberuflich mit diesem Gegenstand befassen, so sind sie doch zugleich, wie Prof. $\mathrm{H}$. Krüger hervorhob, „Multiplikatoren“ und können als solche das Interesse an der Verfassungsentwicklung gerade der Dritte-Welt-Staaten in gewissem Umfang wecken und erweitern. Das gilt um so mehr, als die professionellen „Multiplikatoren" wie etwa die Medien diese Aufgabe nur unzureichend erfüllen.

$\mathrm{Daß}$ dies sogar in einem politisch so aktuellen Bereich wie dem südlichen Afrika zutrifft, zeigten die beiden ersten Länderberichte der Tagung. Während Prof. H. Külz (Berlin) im Anschluß an sein ausführliches Referat auf der Vorjahrestagung von einer erneuten Studienreise nach Namibia berichtete, gab Dr. W. v. Blittersdorff (Hamburg) eine Einschätzung des rhodesischen „internal settlement“. Obwohl in beiden Gebieten die schwarze Bevölkerungsmehrheit an der Schwelle zur politischen Verantwortung zu stehen scheint, die sie mit Ablauf dieses Jahres übernehmen soll, sind deutliche Unterschiede nicht zu übersehen. So ist es durchaus bezeichnend, daß sich im Konstituierungsprozeß Namibias auch nach dem Scheitern der Turnhallenkonferenz gemischtrassige Parteien bilden, die offene Kritik an der südafrikanischen Politik der ethnischen Zersplitterung üben, während die „interne Lösung“ des Ian Smith nicht nur der weißen Bevölkerung eine Sperrminorität im Parlament für die nächsten zehn Jahre sichert, sondern auch Koalitionen zwischen Schwarzen und Weißen untersagt. In keinem der beiden Gebiete wird sich aber, soviel ergab die im übrigen kontroverse Diskussion über die beiden Berichte, der langwierige Dekolonisationsprozeß nach dem aufgestellten Zeitplan vollziehen; darüber hinaus wurden auch Zweifel angemeldet, ob die evtl. bevorstehende Einigung auf die westlichen Vorschläge für Namibia bzw. die interne Übereinkunft in Rhodesien diesen Prozeß überhaupt fördern könne.

Es folgten zwei gerade in ihrer Gegenüberstellung interessante Referate über die US-amerikanische Verfassungsvergleichung (Dr. M. Scheer, LL. M. [Harv.], Hamburg) und die sowjetische Dritte-Welt-Forschung (Dr. O. Luchterhandt, Hilden). Während der erste Referent die praxisorientierten Gründe für die relativ

1 Uber die 1. Jahrestagung berichteten Krüger, NJW 1977, S. 481 f. u. Hernekamp, VRU 1976, S. 291 ff. Zur zweiten Jahrestagung vgl. Külz, in NJW 1978, S. 471. 
intensive Pflege des vergleichenden Verfassungsrechts in den USA und speziell die praktische Forschungsorganisation bzw. das "Forschungsmanagement" an der Harvard University schilderte, gab der zweite Referent einen weitgespannten Uberblick über die sowjetische Literatur zur Verfassungsentwicklung in der Dritten Welt. In der Diskussion wurde zu Recht auf eine deutliche Parallele in beiden Berichten hingewiesen: sowohl die US-amerikanische als auch die sowjetische Forschung gehen von einem Modernisierungskonzept eigener Prägung aus (obwohl gerade sowjetische Wissenschaftler westliche Modernisierungstheorien vehement angreifen), bei dem sie als Entwicklungsziel mehr oder weniger explizit entweder das liberal-demokratische oder das zentralistisch-sozialistische Verfassungsmodell zugrunde legen. In dieser Verknüpfung zwischen Recht und Ideologie bzw. außenpolitischer Strategie ${ }^{2}$ zeigt sich übrigens auch eine Parallele zur Völkerrechtswissenschaft in den beiden Staaten.

Weitere Kurzberichte betrafen die Entwicklung des Föderalismus in Argentinien (P. Malanczuk, Gießen), die Auswirkungen der Energieverteuerung auf die Staaten der Dritten Welt (Dr. W. Schumann, Bad Homburg), sowie die neueren Entwicklungen auf den Philippinen (G. Ahrens, München) und in Pakistan (H. Longerich, Hamburg). Besonders das Referat über den argentinischen Föderalismus und die Entwicklung des Interventionsrechts legte einen strukturellen Vergleich mit Staaten wie Mexiko, Indien und Pakistan nahe.

Einen gewissen Höhepunkt bildete der abschließende Gastvortrag des Baseler Soziologen Prof. P. Trappe über die „Probleme des Dritten Weges in Afrika südlich der Sahara“. Er machte deutlich, daß Verfassungsvergleichung, die nicht bei einem bloßen Institutionenvergleich stehenbleiben, sondern die politisch-reale Gesamtverfassung der Staaten zum Thema machen will, nicht den Juristen vorbehalten bleiben darf, sondern ein beispielhafter Gegenstand für interdisziplinäre Forschungsansätze ist. Der Gastreferent, der zahlreiche Feldstudien in Afrika geleitet hat, ging aus von der Charakterisierung der Entwicklungsstaaten als „soft states" (G. Myrdal) und von der Abwesenheit des nach Th. Geiger entscheidenden Rechtsdurchsetzungsmonopols; dieser Umstand verhindere die Einführung einer zentralen Planwirtschaft, während umgekehrt der Import kapitalistischer Wirtschaftsstile am Fehlen einer Sozialgesetzgebung scheitere. Der „Dritte Weg“ wurde durch das Beispiel Tansanias illustriert, wo die Ujamaa-Dörfer als „natürche Sozialhilfeunternehmungen " fungieren. Neben einem Überblick über die wegweisenden politischen Wertvorstellungen Julius K. Nyereres ${ }^{3}$ enthielt der Vortrag zahlreiche wertvolle Beispiele für Schwierigkeiten und Erfolge im Agrarsektor des Landes, der das Rückgrat seiner Entwicklung bildet. Mit einer angeregten Diskussion der Thesen des Gastreferenten endete die 3. Jahrestagung des Arbeitskreises.

Zuvor waren die Mitglieder übereingekommen, daß die Kommunikation innerhalb des Arbeitskreises, die sich bislang auf die Jahrestagungen beschränkte, durch ein vierteljährliches Mitteilungsblatt verbessert werden sollte. Überdies befürwortete

2 So z. B. die US-Menschenrechtspolitik, mit deren Tradition im Verhältnis zu Lateinamerika sich Prof. K. Krakau in seinem Vortrag auf der Vorjahrestagung des Arbeitskreises beschäftigte (VRO 1977, S. $555 \mathrm{ff} ., 560 \mathrm{ff}$.$) .$

3 Ein vollständiges Verzeichnis der Schriften und Reden Nyereres bis 1969 findet sich in der ideologiekritischen Studie von J. R. Nellis, A Theory of Ideology - The Tanzanian Example, Nairobi 1972 (S. 196 f.); neuerdings Nyerere, Freedom and Development, Dar es Salaam 1973. Allgemein zum Dritten Weg in der 3. Welt, vgl. Krüger VRU 1977, S. 7 ff. 
es eine ganze Reihe von Mitgliedern, die Lüdenscheider Treffen in Zukunft unter ein oder mehrere gegenständliche bzw. strukturelle Themen zu stellen, um auf diese Weise über eine Vielzahl unterschiedlicher Länderberichte hinaus verstärkt zur eigentlichen Verfassungsvergleichung überzugehen. Es wäre ein erster Schritt in dieser Richtung, wenn die Länderreferenten sich bereit fänden, ihre Berichte an gemeinsamen Leitthemen (z. B. Grundrechtsschutz) auszurichten. Die Zusammenarbeit im Arbeitskreis für Überseeische Verfassungsvergleichung hat sich, das zeigte auch die 3. Jahrestagung, so weit entwickelt, daß dieser Schritt gewagt werden sollte.

Alexander Dix 